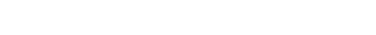

\title{
Les phénomènes gestionnaires à l’épreuve de la pensée économique standard
}

\section{Une mise en perspective de travaux de Jean Tirole}

Dans cet article, I'auteur discute en détail cinq articles de Jean Tirole, prix Nobel d'économie ${ }^{1}$, qui traitent de phénomènes gestionnaires. Il met en évidence les modes de construction de ces articles, évalue la pertinence de leurs résultats de recherche et étudie la réception de ces travaux de recherche auprès des décideurs publics et privés. Il met en perspective ces articles par rapport à une sélection de travaux gestionnaires qui proposent des analyses différentes de ces phénomènes. Il discute enfin les limites de l'approche dite des « faits stylisés » mobilisée par les économistes modélisateurs. 
J ean Tirole, prix Nobel d'économie 2014 et son équipe de la Toulouse School of Economics (TSE) occupent une position prééminente dans le champ économique, comme l'atteste l'impact académique de ses travaux ${ }^{2}$ qui s'inscrivent dans le paradigme, aujourd'hui dominant en micro-économie, de la théorie des incitations et de l'information. Mais l'impact de ses travaux va cependant bien au-delà de la sphère académique : il a également produit de nombreux rapports d'expertise auprès de décideurs publics et privés et parfois influencé l'élaboration des politiques publiques en développant, sous une forme vulgarisée, les résultats de ses travaux modélisateurs. Ainsi, l'ambition théorique se double chez lui (et son équipe) d'une ambition performative : transformer la réalité par des propositions concrètes pour qu'elle devienne plus conforme aux théories économiques. Ce souci de combiner analyse théorique rigoureuse, via des modèles, et ancrage empirique dans des problèmes contemporains s'opère à travers l'approche très particulière de la « modélisation à partir de faits stylisés ».

En quoi consiste cette démarche ? Il s'agit de partir d'un problème concret, décrit de façon simplifiée, qui présente une énigme pour les sciences économiques standard. Des modélisations sont ensuite proposées qui permettent de résoudre cette énigme. Elle se conclut par des préconisations en termes d'incitations ou d'action publique. Remarquons que cette approche est très différente de la recherche dite «mains- tream » en sciences de gestion qui se fonde sur une démarche hypothético-déductive associée à des études économétriques. La modélisation à partir de faits stylisés, également pratiquée en finance, se distingue des recherches mainstream en sciences de gestion sur trois points : les articles n'ont pas de section méthodologique; le mode de validation est uniquement théorique : il porte sur la cohérence et la robustesse de la modélisation; la validation empirique ne se pose pas puisqu'il est admis que les faits stylisés sont, par construction, différents des faits observables et qu'ils remplissent un objectif: décrire un phénomène dans le langage de la micro-économie standard de sorte qu'il se prête à un exercice de modélisation.

Si les travaux de Tirole sont, à ce point, différents de ceux de la plupart des chercheurs en gestion, pourquoi les discuter dans une revue en sciences de gestion ? En premier lieu, parce qu'il a la particularité de s'intéresser à des phénomènes gestionnaires à partir desquels il propose des thèses différentes, parfois orthogonales, de celles des chercheurs en gestion. À ce titre, il semble utile de comprendre en quoi les hypothèses, la démarche de recherche et les résultats présentés par Jean Tirole peuvent différer de ceux des chercheurs en gestion. En deuxième lieu, parce que ses thèses rencontrent un grand succès auprès des décideurs publics et privés et qu'il convient de s'interroger sur les raisons de ce succès. Enfin, parce que bien qu'ayant obtenu une large couverture médiatique suite à son prix

2. Il fait partie des économistes les plus cités au monde avec plus de 90000 citations dans Google Scholar. Sa production scientifique est impressionnante avec près de 500 publications, dont 5 livres de référence et un grand nombre d'articles dans les revues économiques les plus prestigieuses. 
Nobel, les débats de fond demeurent très rares $^{3}$, comme si cette récompense avait anesthésié toute discussion critique 4 .

L'article s'appuie sur la discussion de cinq articles sélectionnés pour leur résonance avec des travaux gestionnaires. Celle-ci ne portera évidemment pas sur la rigueur des modèles et leur robustesse. Non pas que la modélisation ne soit pertinente - elle est évidemment utile pour identifier et caractériser des phénomènes complexes - mais parce que l'analyse des modèles constitue l'activité principale de l'évaluation scientifique dans les revues économiques. Aussi, centrons-nous la discussion sur trois points qui prêtent à une interrogation gestionnaire : - la «fabrication» des recherches : comment les articles sont-ils construits ? Quelle problématisation des phénomènes étudiés propose-t-il ? Quel cadre théorique utiliset-il ? Sur quelles hypothèses se fonde-t-il ?

- les résultats de recherche et leur pertinence : en quoi sont-ils convergents ou divergents avec les travaux en sciences de gestion étudiant les mêmes phénomènes ? Qu'est-ce que la modélisation économique échoue à capturer?

- la réception de ses travaux : pourquoi rencontrent-ils un large écho chez les décideurs publics et privés ?

Dans un premier temps, nous mettons en évidence les modes de construction des articles de Jean Tirole ainsi que les hypothèses fondamentales qui en constituent le soubassement scientifique. Dans un deuxième temps, nous discutons plus en profondeur cinq articles qui ont été sélectionnés parce qu'ils portent sur des phénomènes gestionnaires importants, étudiés par ailleurs par la littérature en sciences de gestion : la régulation des entreprises, leur gouvernance, leur responsabilité sociale et environnementale, les marchés bifaces, le logiciel libre. Pour chacun d'eux, nous présentons d'abord les résultats que nous mettons en perspective par rapport aux résultats produits sur ces questions par la littérature gestionnaire. Enfin, dans un troisième temps, nous analysons la réception des travaux de Jean Tirole et de son équipe de recherche par les décideurs publics et privés.

\section{I - COMMENT LES ARTICLES ACADÉMIQUES DE JEAN TIROLE SONT-ILS CONSTRUITS ?}

\section{1. Épistémologie, théorie et méthodologie}

Les travaux de Tirole se prêtent à une approche synoptique car ils présentent une grande cohérence sur les plans épistémologique, théorique et méthodologique. Il a développé, avec Jean-Jacques Laffont, à la TSE, une école de pensée qui bénéficie d'une aura internationale qui a peu d'équivalents dans le monde des sciences économiques.

Comme il le rappelle dans un article de synthèse paru en 2013 dans la Revue Française d'Économie (Tirole, 2013), son épistémologie se situe clairement dans la lignée de l'économie néoclassique : à savoir celle

\footnotetext{
3. Une exception est l'article écrit par David Encaoua dans la Revue d'économique politique qui présente un portrait laudatif et détaillé des apports de Tirole à la pensée économique (Encaoua, 2015).

4. Il existe, certes, de nombreuses critiques de ce que certains appellent la " théorie économique standard étendue », c'est-à-dire l'ensemble des travaux qui partagent des hypothèses fondamentales sur la rationalité des agents économiques ainsi qu'un cœur normatif commun (voir Favereau, 2006), mais ce courant n'a pas produit de critique spécifique des travaux de Jean Tirole.
} 
d'agents économiques rationnels et individualistes qui cherchent maximiser leur utilité, au sens large.

Sur le plan théorique, il revendique son inscription dans deux cadres théoriques unificateurs : la théorie des jeux et la théorie de l'information (Tirole, 2013). Dans un cas, il s'agit de modéliser les comportements stratégiques d'acteurs confrontés à des jeux répétés qui peuvent être coopératifs ou non. Dans l'autre cas, il s'agit de modéliser des phénomènes d'asymétrie d'information et d'opportunisme dans le cadre de relations principal-agent par le biais de différents outils analytiques: théorie des contrats, théorie du signal, théorie des incitations. La question qui est posée porte sur les moyens dont dispose le principal pour surmonter deux risques identifiés dans la littérature : l'aléa moral (certaines actions de l'agent ne sont pas vérifiables par le principal) et l'antisélection (un agent dispose d'informations qu'il peut dissimuler ou manipuler).

Les modèles visent alors à identifier les systèmes d'incitation les plus adaptés pour éviter les risques d'opportunisme et amener les agents à révéler les informations qu'ils détiennent. Que ce soit dans la théorie des jeux ou la théorie de l'information, les problèmes sont présentés de telle façon qu'ils sont sans ambiguïté et ne peuvent être interprétés qu'au prisme de l'une ou l'autre de ces théories. Cette modélisation sous forme de «faits stylisés » est propre à la théorie économique standard et se distingue des travaux qualitatifs en sciences de gestion où les auteurs visent souvent des descriptions riches des faits empiriques (thick descriptions) qui peuvent être interprétées et traitées selon différents cadres théoriques. Au contraire, ici, tout ce qui est jugé superflu est éliminé de la présentation du contexte. Ainsi, seuls les détails signifiants pour la modélisation sont retenus : 1'univers est constitué de principaux et d'agents et seuls les éléments purement économiques ou modélisables interviennent. C'est ici la cohérence interne de la démonstration qui est recherchée; le degré d'adéquation du récit stylisé avec les situations réelles n'est pas développé.

Sur le plan méthodologique, l'approche de Tirole est modélisatrice. Rares sont ses travaux qui s'appuient sur des études économétriques ou sur de l'économie expérimentale. La conséquence de cette orientation est que, contrairement aux travaux en sciences de gestion où la section méthodologique occupe une bonne partie de l'article afin de justifier la rigueur méthodologique dans le traitement du matériau empirique (qu'il s'agisse de données qualitatives ou quantitatives), les articles de Tirole n'en ont pas. La rigueur scientifique porte avant tout sur la cohérence des modèles tandis que la partie « faits stylisés » ne fait pas l'objet de validation particulière.

Le mode de construction des articles suit une même trame. L'auteur part généralement d'un phénomène empirique stylisé, bien identifié et présenté comme énigmatique pour les sciences économiques, c'est-à-dire où les comportements observés contredisent les prédicats de la théorie (par exemple pourquoi certains marchés proposent des services gratuitement aux clients ?) Le phénomène met en scène des principaux et des agents (ou des clients et des fournisseurs) qui ont des objectifs et des préférences clairement identifiés et qui ont des relations asymétriques entre eux. Le comportement de ces acteurs est modélisé et l'on cherche ensuite à identifier des 
mécanismes d'incitation pouvant influer sur leurs comportements. Enfin, différents systèmes d'incitation sont testés, dans le cadre de ces modèles pour répondre aux problèmes identifiés en amont.

Ainsi, pour résumer, ses articles de recherche partent d'un problème empirique nouveau pour l'économie standard que l'auteur va s'attacher à résoudre à l'aide d'une modélisation suffisamment sophistiquée pour aboutir à des conclusions non triviales, mais néanmoins assez simplifiée pour être discutable par d'autres économistes. Ainsi, le critère de validité de la recherche est d'abord interne à l'économie standard et ne suppose pas de validation externe par d'autres disciplines ou par des praticiens.

Mais l'ambition de Jean Tirole et de son équipe à la Toulouse School of Economics (TSE) ne s'arrête évidemment pas à la production d'articles académiques. Elle se prolonge par des rapports d'expertise, des articles de vulgarisation où la modélisation laisse la place à un argumentaire débouchant sur des préconisations. La visée de ces travaux est donc in fine performative : il s'agit d'établir des recommandations concrètes susceptibles de transformer les politiques publiques. C'est l'objet des rapports d'expertise que nous évoquons dans la troisième partie.

\section{Discussion des hypothèses et du cadre d'analyse}

Les recherches de Jean Tirole et de la TSE se fondent sur un ensemble d'hypo- thèses fondamentales qui sont tenues pour acquises et ne sont donc jamais discutées dans les articles. Quelles représentations implicites véhiculent-elles des phénomènes et des activités gestionnaires ?

La première hypothèse fondatrice, cohérente avec le cadre microéconomique classique, est que les agents sont individualistes et calculateurs et visent à maximiser leur utilité au sens large. Les débats ne manquent pas en sciences sociales à propos de la pertinence de cette hypothèse qui est associée à un individualisme utilitariste. Notons que cette orientation conduit ipso facto à évacuer les analyses systémiques et en termes d'action collective. Dans ce cadre, l'action collective n'a pas de spécificité : elle est le produit de relations interindividuelles; d'où le fait de considérer l'organisation comme un nœud de contrats liant des individus ou des groupes d'individus. Cette hypothèse est problématique pour les travaux en sciences de gestion et en théorie des organisations qui considèrent, dans la tradition des sciences sociales, les phénomènes organisationnels comme irréductibles à cette approche individualiste ${ }^{5}$.

La deuxième hypothèse concerne le comportement des agents économiques ou sociaux : ceux-ci seraient fondamentalement opportunistes, cherchant à tirer avantage des situations et notamment de l'existence asymétries d'information. Constatons que cette hypothèse fixiste est largement démentie par l'histoire de la gestion qui révèle des expériences collectives se fondant sur des hypothèses radicalement oppo-

5. La discussion de cet argument est hors du propos de cet article. Mais il est établi que les fondateurs du champ de la théorie des organisations, comme James March ou Herbert Simon, ont d'emblée pris leurs distances avec cette conception utilitariste et individualiste de l'action collective. Les critiques en sociologie ou en anthropologie ne manquent pas non plus à l'égard du paradigme utilitariste. 
sées : le mouvement des relations humaines, la gestion de projet ou les équipes de production à la japonaise se fondent sur un principe de solidarité, d'engagement dans l'incertain et d'action collective qui fait appel à d'autres ressorts que la recherche opportuniste de l'intérêt individuel.

La troisième hypothèse, corrélative à celle d'opportunisme, porte sur celle de l'existence généralisée de situations d'asymétries d'information. Dans ce cadre, l'enjeu n'est pas de produire des connaissances nouvelles puisqu'on fait l'hypothèse que cellesci sont disponibles ou sont produites de manière privée mais de révéler ces informations par le biais de systèmes d'incitation adéquats afin d'éviter le phénomène d'aléa moral. Sans nier le phénomène d'asymétrie d'information, s'agit-il du cas général ou d'un cas particulier? L'asymétrie d'information correspond à des situations particulières où le cadre de l'action collective est stabilisé, c'est-à-dire où les informations et les règles du jeu sont bien identifiées par les acteurs concernés. Or l'action organisationnelle et managériale est souvent bien éloignée de ce cas de figure. Elle est plutôt marquée par des situations d'incertitude : des acteurs s'engagent, bien souvent, dans une action collective alors que les enjeux, les connaissances et, a fortiori, les intérêts de chacun sont plus ou moins bien connus. C'est une situation que nous avons qualifiée par ailleurs «d'incertitudes partagées » (Aggeri, 1999), c'est-à-dire de la reconnaissance mutuelle par les acteurs en amont d'un projet collectif de l'existence d'incertitudes que l'action collective vise à réduire par des processus d'apprentissage collectifs. L'hypothèse d'asymétries d'information revient ainsi à minorer les phénomènes d'apprentissage et de produc- tion de connaissance, considérés pourtant comme centraux dans la littérature managériale (Nonaka et Takeuchi, 1995 ; Argyris et Schön, 1978). À cet égard, l'hypothèse d'apprentissage collectif nous semble plus générale que celle d'asymétrie d'information; cette dernière ne constituant qu'un cas particulier où les connaissances et les règles du jeu sont connues et stabilisées.

La quatrième hypothèse fondatrice est l'existence généralisée de relations d'agence: chaque agent rend compte à un principal qui lui fixe un mandat et met en place des incitations. En fonction de ces incitations, les agents économiques ou sociaux orientent leurs comportements dans un sens ou dans un autre. Logiquement, l'activité gestionnaire se limite, dans cette optique, à l'élaboration de systèmes d'incitation dans le cadre de relations d'agence. Ainsi, dans les organisations, les managers mettent en place des systèmes de motivation pour les salariés. Ces managers sont eux-mêmes évalués en fonction de leur performance par des responsables de business units qui rendent des comptes à leurs dirigeants qui, eux-mêmes, sont soumis aux systèmes d'incitation mis en place par les actionnaires. Cette représentation $\mathrm{du}$ fonctionnement des organisations n'est pas sans rapport avec leur fonctionnement réel. On constate, en effet, depuis vingt ans une convergence entre la théorie et les pratiques, ou dit autrement, une performativité croissante des théories économiques et financières qui ont conduit à modifier en profondeur la représentation de la performance dans les organisations dans un sens proche de la théorie des incitations.

Pourtant cette réalité ne saurait occulter que la gestion des grandes organisations et des entreprises recouvre bien d'autres activités 
que la conception de systèmes d'incitations. Historiquement, les premières doctrines de gestion ont même émergé contre l'inefficacité de systèmes d'incitation de type marchand. C'est en effet contre le système du salaire des ouvriers aux pièces tel qu'il existait au XIX ${ }^{\mathrm{e}}$ que Taylor a proposé un mode d'organisation radicalement nouveau fondé sur des relations de prescription qui a profondément modifié les rapports entre conception et exécution du travail (Hatchuel, 1994). De même, les principes de la direction fayolienne introduisent tout un ensemble de principes qui fondent une science administrative qui n'est en aucune manière réductible à des règles d'incitation (Peaucelle, 2003). Quant à la théorisation de Barnard du "common purpose » comme fondement de l'action collective et des processus de coopération, elle est orthogonale avec la théorie de l'agence (Barnard, 1938). D'autres doctrines de gestion plus récentes, comme les systèmes de gestion japonais (qualité totale, juste-à-temps, progrès continu), qui ont fait largement la preuve de leur efficacité, se fondent sur d'autres représentations de la théorie de l'agence, le travail en équipe et la solidarité notamment (Nonaka et Takeuchi, op. cit.). Enfin, depuis vingt ans, des travaux en sciences de gestion proposent de considérer la stratégie comme science de la conception et la gestion comme ingénierie (voir Martinet et Pesqueux, 2013 ; Hatchuel, 2012). Dans cette perspective, l'activité stratégique et gestionnaire consiste à inventer de nouveaux modèles de management et de nouvelles instrumentations de gestion, à conduire des expérimentations et des explorations dans l'inconnu qui ne sont pas connaissables a priori ; toutes activités qui ne sont pas pensables dans le seul cadre de la théorie des incitations et de l'agence ni réductibles à ces questions.

$\mathrm{Au}$ final, l'ensemble des hypothèses sur lesquelles se fondent les travaux de Jean Tirole, et avec lui de la microéconomie standard, dessine, en creux, une représentation réductrice des phénomènes gestionnaires : ceux-ci se résument à la conception de systèmes d'incitation dans le cadre de relations principal-agent où il s'agit d'éviter les risques d'opportunisme des agents (firmes ou individus).

\section{II - COMMENT JEAN TIROLE TRAITE-T-IL DE PHÉNOMÈNES GESTIONNAIRES ?}

Après l'examen du mode de construction des articles, venons-en à une discussion plus précise de certains articles que nous avons sélectionnés parce qu'ils portent sur des phénomènes gestionnaires importants, étudiés par ailleurs par la littérature en sciences de gestion: les marchés bifaces, le logiciel libre, la gouvernance des entreprise, la RSE et les stratégies politiques des entreprises. Pour chacun d'eux, nous présentons d'abord les résultats proposés que nous mettons en perspective par rapport aux résultats produits sur ces questions par la littérature gestionnaire.

\section{De nouvelles stratégies de création de valeur : concurrence sur les plates- formes et marchés « bifaces »}

Jean-Charles Rochet et Jean Tirole ont écrit plusieurs articles célèbres sur un phénomène énigmatique : les marchés bifaces structurés autour de plates-formes (Rochet et Tirole, 2003). Le point de départ de leur analyse est l'existence d'une plate-forme qui permet l'interaction entre deux types d'utilisateurs situés des deux côtés de celle- 
ci. Le marché est dit biface si la tarification supplémentaire sur l'une des faces de la plate-forme affecte le volume des transactions sur l'autre face. On dit alors qu'il y a des externalités croisées entre ces deux marchés. Ce mécanisme permet d'expliquer pourquoi il est possible d'avoir des biens et services vendus gratuitement sur une face dès lors que ce manque à gagner est compensé par les prix demandés sur l'autre face. Un tel exemple est à l'œuvre dans les journaux gratuits. Le journal constitue une plate-forme avec deux types d'utilisateurs : des lecteurs, qui retirent une utilité de la lecture, et des annonceurs, qui sont prêt à payer en fonction du nombre de lecteurs. La stratégie des « gratuits » a été de réduire à zéro le prix de vente pour attirer un plus grand nombre de lecteurs et d'augmenter, en parallèle, le prix des encarts publicitaires en s'appuyant sur un consentement à payer plus élevé des annonceurs. Les systèmes de paiement (cartes de crédit) avec deux types d'utilisateurs (commerçants et titulaires des cartes) qui utilisent la «plateforme » (la carte de crédit) constituent un autre exemple de ce type. Plus largement, on trouve un grand nombre de marchés bifaces autour des nouvelles technologies de l'information et de la communication ou dans le domaine des jeux vidéos; les consoles constituant les plates-formes, les joueurs et les développeurs de jeux étant alors les utilisateurs de celles-ci.

Rochet et Tirole analysent les mécanismes de formation des prix sur ces marchés qu'ils considèrent comme la question centrale. Ils analysent les externalités de réseaux considérés et les élasticités croisées entre les deux marchés, et évaluent différentes stratégies de tarification s'appuyant sur des coûts d'abonnement (visant à couvrir les coûts fixes) et des tarifications liés à l'usage. Ils proposent, à partir de cette analyse, un modèle général de formation des prix sur les marchés bifaces.

\section{Discussion}

Ce même phénomène a été étudié selon un prisme différent par des chercheurs en gestion. Annabelle Gawer et Michael Cusumano, spécialistes de l'innovation sur les plates-formes, soulignent les limites des travaux de Tirole et Rochet : les questions de tarification leur semblent de second ordre par rapport aux enjeux d'innovation sur les plates-formes et aux nouveaux business models qui y sont associés (Gawer et Cusumano, 2010). Pour ces auteurs, l'enjeu stratégique pour les entreprises est de concevoir des plates-formes et de construire un écosystème d'affaires autour de celles-ci. C'est l'activité » qui est de loin la plus difficile et la plus coûteuse. Y parvenir avant les concurrents permet de constituer une rente temporaire pour les innovateurs. En outre, plus que le prix en lui-même, c'est la proposition de valeur qui est critique dans les business models des plates-formes pour capter et fidéliser des clients. Une fois ce travail de conception effectué, l'élaboration d'un système de tarification peut être établi. Mais, pour ces auteurs, c'est un problème technique secondaire qui ne présente pas de difficultés particulières.

\section{Les régimes d'open innovation : le cas du logiciel libre}

Autre phénomène énigmatique d'innovation ouverte : l'open source ou le logiciel libre. Pourquoi des programmeurs participent-ils à des projets collectifs et à la production de biens publics? Comment expliquer cet engagement alors qu'ils ne 
sont pas directement rémunérés pour ces activités et qu'ils ne peuvent en tirer de bénéfices en termes de droits de propriété ? Josh Lerner et Jean Tirole proposent une modélisation de ce phénomène à partir de l'idée que les programmeurs visent en fait, à travers cette activité, à accroître leurs compétences afin d'améliorer leur réputation grâce à la répétition des projets et, in fine, à améliorer leur employabilité et leurs carrières (Lerner et Tirole, 2002). C'est la théorie du signal : plus la performance est visible auprès du public, plus la contribution individuelle au projet collectif est visible, plus la performance informe sur la qualité des talents, plus forte sera l'incitation pour les programmeurs de participer à ces projets. Ainsi, pour Lerner et Tirole, le mécanisme fondamental au cœur de l'open source serait donc lié à des enjeux de motivations individuelles. Ils notent également que les entreprises peuvent avoir intérêt à participer aux processus en proposant des services payants complémentaires aux logiciels libres.

\section{Discussion}

Comment les spécialistes de gestion interprètent-ils le même phénomène ? La plupart des auteurs s'interrogent sur les dynamiques collectives qui sous-tendent le développement de l'open source (Von Krogh et Von Hippel, 2003). Ils soulignent d'abord que le régime de l'open source correspond à un nouveau régime d'action fondé sur la participation active d'utilisateurs (end users) qui deviennent concepteurs (Benkeltoum, 2011). Le sentiment d'appartenance à une communauté semble constituer une motivation extrêmement forte, d'autant plus que les développeurs sont également des utilisateurs et qu'ils valorisent fortement le fait de participer à ces projets collectifs et les occasions d'apprentissages que favorisent ces derniers. La motivation individuelle ne semble donc pas se réduire à un calcul d'intérêt, même si celui-ci existe, mais doit prendre en compte le besoin d'adhésion et d'engagement dans des projets collectifs et des communautés d'acteurs qui partagent une certaine vision de la conception de biens communs.

Certains auteurs prolongent, d'un point de vue stratégique, les développements de Lerner et Tirole sur les complémentarités entre logiciel libre et fermé. Ainsi, certaines entreprises privées cherchent à conduire des stratégies de verrouillage en produisant des biens privés à partir de ressources collectives (Von Krogh et Von Hippel, 2003). Les firmes s'impliquent dans l'open source à la fois pour améliorer la compatibilité entre logiciels libres et fermés et pour promouvoir l'interopérabilité entre systèmes.

Enfin, d'autres auteurs s'intéressent aux processus de conception dans ces régimes d'open source: comment des processus de conception apparemment horizontaux convergent-ils vers un ensemble de solutions ? Certains auteurs soulignent également que loin d'être un processus purement horizontal des hiérarchies émergentes sont associées à la conception du logiciel libre ainsi que la production de normes, de règles et de langages communs qui permettent de structurer l'action collective (Benkeltoum, 2011).

\section{La gouvernance des entreprises}

Dans un article de 2001 sur la corporate governance, Jean Tirole examine une analyse économique du concept de shareholder value et propose une première analyse du concept de société partenariale (stake- 
holder society). La définition standard de la gouvernance d'entreprise, rappelle-t-il, s'appuie sur deux principes: la défense des intérêts des actionnaires, d'une part, la séparation de la propriété de l'entreprise et du contrôle, d'autre part. Dans la lignée des travaux sur la théorie de l'agence et les droits de propriété (Grossman et Hart, 1986), Tirole considère le modèle actionnarial comme la conception de relations principal-agent avec, au sommet, des propriétaires actionnaires qui en tant que créanciers résiduels (residual claimants) prennent des risques particuliers par rapport aux autres créanciers et bénéficient à ce titre du pouvoir de contrôle sur les managers qui leurs délèguent des droits de contrôle. Dans cette perspective, les actionnaires sont les principaux et sont en droit d'exiger des dirigeants (leurs agents) de maximiser la valeur actionnariale de l'entreprise. De ce postulat découle toute une série de préconisations en matière de bonne gouvernance. Tirole examine d'abord la performance de ce modèle à l'aune de la théorie des incitations. Il souligne que ce modèle est davantage susceptible que d'autres d'augmenter les revenus de l'entreprise, de focaliser l'attention des managers sur un critère simple et de définir une structure de contrôle claire. Il présente toutefois un inconvénient : orienter les décisions dans le seul intérêt des actionnaires.

Il étudie ensuite une hypothèse rivale : une gouvernance fondée sur un principe partenarial où les dirigeants seraient redevables à l'égard de parties prenantes. Il met en évidence les limites d'un tel modèle partenarial : la maximisation du chiffre d'affaires est moins garantie ; il existe un risque de dispersion des managers confrontés à des incitations multiples; le partage du contrôle entre stakeholders, une sorte de codétermination généralisée, même s'il s'agit d'un mode de décision plus démocratique, est susceptible de produire des effets pervers sous la forme d'une paralysie de la décision et de comportement de passager clandestin. Il conclut à la supériorité opérationnelle du modèle actionnarial, notamment en termes de contrôlabilité, même si des enjeux politiques légitimes pourraient justifier une ouverture vers les parties prenantes.

\section{Discussion}

De nombreux travaux en gestion se sont intéressés à l'émergence d'un modèle actionnarial et à celle d'un modèle partenarial. Comment problématisent-ils ces sujets ? Quels sont les points de divergence avec l'article de Tirole? Ceux-ci portent d'abord sur le cadrage initial. Dans cet article, les actionnaires seraient les principaux et les dirigeants seraient leurs agents. Ce constat supposé, formulé comme une évidence, est discutable à la fois sur le plan historique et normatif. Il a été contesté par de nombreux juristes qui font valoir que les économistes méconnaissent le raisonnement juridique. En droit, les actionnaires ne sont pas propriétaires de l'entreprise mais uniquement de leurs actions. La société anonyme (corporation), personne morale, est une construction juridique complexe qui a été conçue afin d'éviter un contrôle exclusif au profit des seuls actionnaires (voir Blair et Stout, 1999). Elle est, en effet, dotée d'un objet social que les associés sont tenus de respecter. Les dirigeants ne sont pas les mandataires des actionnaires mais ceux de la société anonyme. Ainsi, en cas de conflit entre des actionnaires minoritaires et les managers de l'entreprise, les actionnaires doivent démontrer que les managers ont agi 
contre l'intérêt de la société anonyme (et non pas seulement contre leur intérêt). Ce n'est qu'à cette condition que le juge peut éventuellement trancher en leur faveur. En aucun cas, l'argument de la maximisation de la valeur actionnariale ne peut suffire. Quant à la révocation des managers par les conseils d'administration, ce ne sont pas directement les actionnaires qui le font mais leurs mandataires qui siègent au conseil d'administration. Pour ce faire, un débat doit avoir lieu au préalable et un vote doit être organisé.

Ainsi, les juristes rappellent que la construction du droit des sociétés ne correspond pas au modèle de la théorie de l'agence. La société anonyme a été formée comme un dispositif équilibré qui vise d'une part, à limiter la responsabilité des actionnaires vis-à-vis de manœuvres discutables des managers et d'autre part, à protéger, grâce à des règles procédurales précises, les managers de l'arbitraire des décisions des actionnaires. Pour qualifier cette logique juridique et le fonctionnement des entreprises, Margaret Blair et Lynn Stout proposent de remplacer le modèle de l'agence par celui de l'équipe de production (team production) qui qualifie mieux, selon elles, le projet collectif de l'entreprise comme étant constitué de solidarités et d'engagements réciproques matérialisés dans le dispositif juridique de la société anonyme.

Les travaux en sciences de gestion ont également critiqué la théorie de l'agence appliquée à la gouvernance d'entreprise, notamment celui de Tirole présenté plus haut. Ils contestent d'abord l'idée même d'un modèle actionnarial clairement identifiable. Pierre-Yves Gomez souligne que le développement de l'actionnariat de masse et la montée des investisseurs institutionnels ont profondément modifié le jeu du gouvernement d'entreprise. On observe une hétérogénéité croissante des intérêts en présence et de leurs temporalités (Gomez, 2003) et nulle convergence vers une hypothétique maximisation de la valeur actionnariale dont Gomez relève qu'il s'agit davantage d'une construction idéologique que d'un concept scientifiquement et empiriquement fondé. Il souligne ensuite que le pouvoir discrétionnaire du dirigeant ne se joue pas uniquement dans des relations d'agence avec l'actionnaire mais se règle à travers des règles institutionnelles et juridiques qui font intervenir une multiplicité de parties prenantes. Ainsi, plusieurs auteurs ont observé la montée des parties prenantes dans la gouvernance des entreprises et proposent des pistes pour fonder un modèle partenarial (Charreaux et Desbrières, 1998). $\mathrm{Si}$, dans certaines entreprises, les dirigeants se comportent comme si les actionnaires étaient propriétaires c'est donc moins parce que la loi les y incite que parce qu'ils ont intériorisé cette norme dans l'évaluation de leurs performances sous la pression de groupes d'intérêts ou mus par la crainte d'être démis de leurs fonctions. Il n'y a pourtant pas de fatalité à ce que toutes les sociétés anonymes fonctionnent ainsi. Dans cette perspective, les travaux récents de Blanche Segrestin et d'Armand Hatchuel ont cherché à aller plus loin dans la modélisation de l'entreprise comme projet stratégique collectif qui se concrétise à travers une mission (Segrestin et Hatchuel, 2012). En formalisant davantage cette mission dans un objet social étendu, les actionnaires potentiels seraient alors tenus par des engagements concernant le respect de cette mission et auraient davantage de difficultés à le distordre à leur profit. Contrairement à la 
théorie de l'agence qui fait l'hypothèse que l'objet social de l'entreprise est donné, les travaux en droit et gestion mettent ainsi en évidence que celui-ci est au cœur de la stratégie des entreprises. Pierre-Yves Gomez souligne que le développement d'autres formes juridiques des grandes entreprises que la société anonyme (mutuelles, coopératives, fondations, etc.) met en évidence qu'il existe bien des manières d'échapper au modèle actionnarial sans obérer pour autant les capacités de financement et de développement (voir Gomez, 2003). Il est à noter que ces autres formes fonctionnent selon des modèles et des hypothèses radicalement différentes de celles que Lerner et Tirole ont de la théorie de l'agence. Pour résumer, la modélisation qu'ils proposent ne rend pas compte de la façon dont la société anonyme a été constituée dans le droit des sociétés et elle ne rend pas davantage compte d'autres formes de société que celles de la société anonyme. Par ailleurs, l'alternative discutée entre modèle actionnarial et partenarial semble assez théorique et ne correspond pas aux choix de gouvernance que font les entreprises. Lorsque d'autres parties prenantes que les actionnaires, comme les salariés, sont représentés au conseil d'administration leur participation s'explique généralement par l'existence, comme en Allemagne, d'un cadre juridique et institutionnel différent fondé sur la codétermination (mit bestimmung). Quand les entreprises ont le choix de leur gouvernance, elles ont la possibilité d'adopter d'autres formes juridiques que celle de la société anonyme (coopératives, SCIC, SCOP, mutuelles, etc.) si elles souhaitent intégrer d'autres parties prenantes que les seuls actionnaires.

\section{L'entreprise dans la société : le débat sur la RSE}

Jean Tirole s'est également intéressé, avec Roland Benabou, au phénomène de la RSE (Benabou et Tirole, 2010). Les auteurs rappellent que la théorie économique standard considère que la société devrait être organisée sur deux piliers : la main invisible des marchés et la correction des défaillances de marché par l'État. L'entreprise étant censée maximiser la valeur pour l'actionnaire, les stakeholders sont protégés par des contrats et par la réglementation. Les auteurs examinent les demandes croissantes de la société pour une responsabilité sociale des individus et des entreprises comme une réponse alternative aux défaillances du marché et à celles de l'action publique. Ils indiquent qu'il ne faut pas négliger ce mouvement de la RSE qui devient significatif avec la montée de l'action des ONG, l'accessibilité croissante aux informations extra financières sur les entreprises et l'augmentation du coût à long terme des pollutions, notamment atmosphériques.

Les auteurs reprennent la définition classique de la RSE en économie qui peut être formulée comme le fait de sacrifier des profits pour un intérêt social, c'est-à-dire d'aller au-delà des obligations réglementaires sur une base volontaire.

À cette aune, la question que se posent les auteurs est la suivante: pourquoi les citoyens et les entreprises se donnent-ils pour mission d'agir dans ces domaines et de se substituer aux gouvernements élus ?

La première réponse avancée tient aux défaillances de la puissance publique qui peut être capturée par les intérêts privés, agit dans le cadre de juridictions territoriales alors que les entreprises globalisées 
agissent dans un espace mondial et font face à des coûts de transaction élevés. La réponse tient à ce que les agents souhaitent promouvoir des valeurs qui ne sont pas partagées par les décideurs publics.

Les auteurs se lancent ensuite dans une discussion des facteurs qui peuvent expliquer les comportements prosociaux des individus ou des entreprises. Les auteurs distinguent notamment les motivations intrinsèques (altruisme) de motivations extrinsèques (liées par exemple à des incitations monétaires). S'appuyant sur des travaux de psychologie expérimentale, ils soulignent l'importance de l'image publique et de l'image de soi comme facteurs explicatifs de ces comportements. Toutefois, ils mettent en évidence les risques de justification excessive (overjustification) qui peuvent conduire les destinataires de ces comportements à les attribuer non pas à de l'altruisme mais à la recherche d'image. Les auteurs soulignent qu'il importe, d'un point de vue psychologique, que les signaux soient interprétés de sorte que les entreprises soient perçues comme n'étant pas concernées uniquement par leur image. Ils passent ensuite en revue trois conceptions classiques de la RSE : la théorie du gagnant-gagnant qui considère qu'il faut dépasser le court termisme pour intégrer les gains à long terme d'une approche RSE; la demande pour que les entreprises agissent au nom des stakeholders en matière de philanthropie (comme dans le cas du commerce équitable); la philanthropie d'entreprise fondée sur les convictions personnelles des dirigeants. Les auteurs discutent chacune de ces conceptions en en montrant les limites et s'interrogent en conclusions sur le fait que ces initiatives constituent des compléments ou des substituts à l'action publique.

\section{Discussion}

Il est indéniable que certains mécanismes identifiés par les auteurs ont joué un rôle dans le développement de la RSE comme dans la montée en puissance des capacités d'action de la société civile, les défaillances croissantes de l'action publique ou les enjeux d'image. Il est toutefois frappant de constater que les auteurs cherchent à fournir des explications individuelles et ahistoriques à un phénomène, dont certains ont analysé l'historicité et les enjeux collectifs et institutionnels.

La RSE n'est pas un phénomène nouveau. Elle a émergé dans un contexte précis aux États-Unis qui correspond à la fois à une crise de légitimité de l'entreprise et de l'action publique et une revendication forte de nouveaux groupes concernés issus de la société civile. Ses formes contemporaines doivent être analysées dans le cadre d'un phénomène de mondialisation et de globalisation des firmes dotées d'un pouvoir sans précédent. On peut interpréter le mouvement de la RSE comme une contestation de ce pouvoir croissant et une interpellation de différentes parties prenantes concernant les responsabilités sociales et environnementales des entreprises, des plus grandes en particulier (Capron et Quairel, 2015). Dans cette perspective, l'entreprise constitue une institution politique qui est cœur des enjeux de société contemporains (Martinet, 1984). Mais il est à noter que le mouvement de la RSE ne revêt pas les mêmes formes partout. Les dimensions institutionnelles, absentes dans l'article de Benabou et Tirole, jouent un rôle central. Dirk Matten et Jeremy Moon ont montré, par exemple, que ce qui est considéré comme implicite en Europe où les exigences réglementaires sont plus 
fortes (ex. : la formation, la gestion des compétences) sont considérées comme faisant explicitement partie de la RSE aux États-Unis; cette frontière entre implicite et explicite évoluant au fil du temps en fonction de l'évolution du cadre légal et réglementaire (Matten et Moon, 2008). Cette analyse fait ressortir que la RSE ne saurait se limiter à la théorie d'une action purement volontaire. Elle consiste d'abord à anticiper des menaces et gérer une contestabilité (Adant et al., 2005) avant qu'elles se transforment en crise de légitimité et en risque réputationnel qui peuvent, à leur tour, justifier une intervention publique. Dans cette même logique d'anticipation, la rhétorique du win-win, proposée par certains auteurs (voir Porter et Kramer, 2006), est simpliste. Les stratégies d'innovation en matière de RSE sont des paris dans l'inconnu. Certaines entreprises pionnières s'engagent dans ces voies, malgré les incertitudes, parce qu'elles estiment que leur activités et leurs marchés actuels sont menacés à plus ou moins longue échéance par les effets environnementaux et sociaux que leurs activités engendrent (Aggeri et al., 2005). Là encore, la recherche de nouveaux modèles économiques et la conduite de projets d'innovation ne sont pas purement volontaires et se trouvent étroitement liées à de nouvelles politiques publiques susceptibles de créer un environnement plus favorable à ces projets, comme dans le cas des véhicules propres. À cet égard, la RSE ne s'oppose pas à l'action publique; les deux s'inscrivent dans un jeu dynamique que les pouvoirs publics, comme les entreprises, cherchent le plus souvent à susciter.

Quant à la question de l'image, c'est, en effet, un enjeu clé. Mais celui-ci se pose de façon différente pour les individus et pour les entreprises. Face à une crise de légitimité sans précédent, les entreprises ont développé des activités de communication et une mise en scène de leurs actions pour donner une image appropriée d'ellesmêmes. Dans ces stratégies, à côté de motivations intrinsèques, ce que font les concurrents (le mimétisme) ou ce que préconisent les consultants (la prescription) (voir Gond et Brès, 2014) constituent des mécanismes clés dans l'adoption de pratiques managériales. Ainsi, la dynamique collective se nourrit non seulement de motivations individuelles mais également de processus collectifs et institutionnels (mimétisme, prescription).

\section{Stratégies politiques des entreprises et régulation}

Jean Tirole a écrit il y a plus de vingt ans une série d'articles avec Jean-Jacques Laffont sur la régulation des entreprises et leurs stratégies politiques (Laffont et Tirole, 1991). Le point de départ de l'analyse était de raffiner les travaux développés par Stigler et d'autres économistes sur les risques de "capture réglementaire » des pouvoirs publics par les intérêts privés (Stigler, 1971). S'appuyant sur des travaux de sociologie et de sciences politiques, la théorie de la capture réglementaire fait l'hypothèse que les agents étant opportunistes, ils cherchent à maximiser leur utilité. Alors que les travaux d'économie publique considéraient à l'origine que les pouvoirs publics étaient garants de l'intérêt général et visaient à maximiser le bien-être social, les travaux sur la capture estiment que les élus sont soumis à des actions de lobbying et cherchent d'abord à défendre leur intérêt propre, à savoir obtenir leur réélection. Modélisant les relations d'agence entre 
pouvoirs publics et industriels, ces travaux ont mis en évidence que les risques de capture sont d'autant plus élevés que les agents (ici les entreprises) sont peu nombreux (oligopole) et capables d'organiser un lobbying efficace. Ces travaux permettent ainsi d'expliquer des situations observées où l'action publique s'éloigne à l'évidence de la protection de l'intérêt général. Par rapport à ces travaux initiaux, Laffont et Tirole ont rajouté un degré supplémentaire de sophistication : les entreprises, qui cherchent à éviter les coûts de la réglementation, dépendent de principaux (les agents administratifs) qui cherchent à maximiser leur carrière qui, à leur tour, dépendent d'élus qui cherchent à être réélus. Dans ce système à trois étages, les auteurs modélisent les asymétries d'information et tous les risques de capture possibles. Ainsi, les entreprises peuvent essayer de s'assurer le soutien des agents administratifs en leur promettant des carrières dans le privé; les élus peuvent être également soudoyés ; enfin, le contrôle des élus sur leurs agents peut s'avérer défaillant. Dans ce contexte, les auteurs imaginent des systèmes d'incitation visant à limiter les risques de capture à ces différents niveaux. Parmi les solutions imaginées pour éviter ces risques, les auteurs proposent un contrôle des agents administratifs par deux tutelles différentes ou bien la surveillance des élus par des autorités indépendantes.

\section{Discussion}

Peut-on réduire les relations entre entreprises et régulateurs à ces jeux d'influence ? Ceux-ci existent et il n'est pas question de les nier. Mais d'autres phénomènes sont à l'œuvre et leur mise à jour requiert d'autres cadres d'analyse. L'un des phénomènes mis en évidence par la littérature en gestion est de considérer que les politiques publiques peuvent constituer un soutien aux stratégies d'innovation des entreprises (Porter et Van der Linde, 1995 ; Aggeri, 1999). Dans le domaine de l'environnement, Michael Porter et Claas Van der Linde indiquent que l'action des entreprises est difficilement concevable en dehors de régulations publiques qui fixent des objectifs ambitieux et permettent de solvabiliser des stratégies environnementales permettant de les atteindre. Contrairement à une idée acquise, les entreprises n'ont pas toutes intérêt à s'opposer systématiquement à la réglementation. Les pionniers peuvent espérer, grâce à la réglementation, acquérir une position stratégique avantageuse et soutenir l'action publique. Pour y parvenir, les entreprises déploient une variété de tactiques et de stratégies qui sont à la fois individuelles et collectives, car elles nécessitent notamment la construction de règles du jeu (normes, standard, langages).

De façon réciproque, les pouvoirs publics ont souvent intérêt à organiser cette course à l'innovation afin de s'assurer le soutien des entreprises pionnières. N'étant généralement pas en capacité d'imposer des standards contraignants aux entreprises, faute de connaissance et de légitimité, les pouvoirs publics cherchent moins à prescrire qu'à orienter, stimuler et accompagner les entreprises vers des stratégies d'innovation (Aggeri, 2000). Dans ces politiques de l'innovation, les phénomènes de capture sont souvent de second ordre par rapport aux enjeux d'innovation et de divulgation des connaissances produites pour permettre de favoriser des dynamiques collectives. 


\section{Synthèse}

Quels points saillants ressortent de la discussion de ces différents cas ? En premier lieu, la description stylisée des phénomènes empiriques est délibérément simple et pointe implicitement vers des mécanismes individuels et d'incitation. Ainsi, toutes les explications collectives, institutionnelles ou organisationnelles sont laissées de côté. Ce n'est pas le principe de la stylisation qui est ici en cause. Il peut être constituer un principe pédagogique efficace pour identifier des mécanismes dans des phénomènes complexes et pour permettre l'exercice de modélisation. Ainsi, certains faits stylisés ont permis de mettre à jour des phénomènes méconnus ou inattendus et d'illustrer des avancées théoriques comme l'illustre l'article célèbre du marché des lemons de Georges Akerlof qui a permis de conceptualiser le phénomène de la sélection adverse (Akerlof, 1970). Mais dans les exemples précédemment étudiés, la stylisation sert surtout à éliminer toutes les explications alternatives plausibles et à justifier les présupposés de la théorie. L'absence de réfutation empirique rend alors tout débat impossible, hors les enjeux de modélisation. Deuxièmement, les problèmes sont posés sans référence à leur historicité. Les conditions historiques dans lesquelles ces problèmes ont émergé sont rapidement décrites ou évacuées. Troisièmement, les acteurs sont dotés d'une rationalité substantive qui n'est influencée que par leur psychologie ou leur essence sans référence aux situations de gestion, à leurs trajectoires individuelles ou aux collectifs auxquels ils appartiennent. Quatrième point qui découle logiquement des trois précédents : les préconisations portent sur la conception de systèmes d'incitation.

\section{III - LA RÉCEPTION DES TRAVAUX DE JEAN TIROLE CHEZ LES DÉCIDEURS}

Quelle est la réception des travaux de Jean Tirole en dehors des cercles académiques ? Hormis la période très récente qui a succédé à son prix Nobel, les médias se sont peu intéressés à ses travaux. Il en va, en revanche, différemment des décideurs publics et privés qui sont extrêmement friands de ce type d'analyse. Le Conseil d'analyse économique, la Commission européenne, certaines entreprises privées (notamment celles qui soutiennent la TSE) font régulièrement appel à ses services sur des thèmes aussi variés que la protection de la propriété intellectuelle, la régulation du commerce ou la réglementation de différents secteurs industriels. Dans les rapports d'expertise, les équations mathématiques disparaissent. Subsistent la description d'un problème politique sous forme de faits stylisés ainsi que des recommandations fondées sur la théorie des incitations et de l'information.

Comment expliquer le succès obtenu par Jean Tirole auprès des décideurs ? Quatre hypothèses principales peuvent être avancées.

En premier lieu, il ne faut pas minimiser le prestige et la légitimité que confère le profil de l'économiste modélisateur revendiquant un ancrage dans la science normale. C'est le vieux principe wébérien de la légitimité rationnelle-légale qui fonctionne toujours : les thèses et les propositions sont d'autant plus légitimes qu'elles s'appuient sur des approches réputées scientifiques. Cette scientificité est pourtant le plus souvent difficile à établir. Les articles précédemment analysés sont conçus de telle sorte que la réfutabilité des arguments n'est possible 
qu'à l'intérieur d'un système de référence dont nous avons montré les limites. Il n'existe pas de réfutabilité empirique possible puisque les faits étant stylisés, ils n'ont pas pour fonction de décrire des faits réels. Si ces recherches étaient purement spéculatives, cela ne poserait pas de problème. Il en va différemment dès lors qu'elles ont un lien avec des prescriptions concrètes. Or les rapports d'étude en question éliminent les modèles mathématiques pour conserver la présentation des faits stylisés et les recommandations. Tout se passe alors comme si ces analyses étaient empiriquement fondées.

En deuxième lieu, il faut souligner la perméabilité croissante des milieux de l'expertise et de la recherche. La réceptivité de ces travaux académiques est d'autant plus grande que les décideurs en question ont souvent une formation économique et que les principes et hypothèses de la microéconomie standard constituent l'alpha et l'oméga des acteurs en charge de la régulation des marchés et de la concurrence. Cette perméabilité constitue un puissant facteur de la diffusion des idées des économistes auprès des décideurs. Elle contribue indéniablement à la performativité des sciences économiques, c'est-à-dire au fait que les théories contribuent à modifier la réalité par le biais de toute une série d'interventions publiques (Muniesa et Callon, 2008). Le projet de transformer la société conformément aux théories économiques est ainsi un objectif partagé par de nombreux acteurs du système économique pour qui la gouvernance néolibérale suppose d'abord de modifier les règles du jeu économique pour transformer les acteurs en agents calculateurs. Dans ce processus, les modèles n'ont pas toujours une fonction performative. Ils peuvent avoir une fonction de légitimation scientifique des propositions. Les faits stylisés ont, en revanche, un rôle clé dans le système argumentatif. Par l'effet de réel qu'ils produisent, ils polarisent, en effet, les débats et donnent une dimension empirique aux préconisations établies. Ils constituent en cela un puissant mécanisme de performation qui est pourtant peu pris en compte par les travaux de sociologie économique sur la performativité des sciences économiques. En troisième lieu, les thèses économiques sont d'autant plus écoutées qu'elles sont formulées simplement et qu'elles désignent des leviers facilement actionnables. La conception de systèmes d'incitation fait désormais partie de la boîte à outils du régulateur qu'il actionne en toute occasion en modifiant tel ou tel potentiomètre. Lorsque le résultat n'est pas à la hauteur, la réponse de l'expert économiste est généralement de considérer que la mise en œuvre de la politique n'est pas conforme aux prescriptions de la théorie. Ainsi, en va-t-il des marchés du carbone qui présentent des dysfonctionnements patents (prix proche de zéro, fraudes, effets d'aubaine). Pour Jean Tirole, qui a produit un rapport sur le sujet, la raison de ces dysfonctionnements tient au fait que la conception de ces marchés est éloignée des propositions théoriques en matière d'allocation des droits à polluer. En revanche, les effets concrets du système comptable et bureaucratique qui sous-tend ces marchés sont rarement questionnés ${ }^{5}$. 


\section{CONCLUSION}

Les travaux de Jean Tirole sont emblématiques d'une certaine façon de considérer la modélisation économique pour produire des analyses et des prescriptions à visée performative. C'est la légitimité de cet objectif d'actionnabilité des modèles économiques qui interpelle les chercheurs en gestion. Si ces derniers visent aussi cet objectif il y a de fortes différences entre les raisonnements et les approches mobilisées. La différence tient au rapport à la théorie et à l'empirie. La micro-économie présente la caractéristique de s'appuyer sur un petit nombre d'hypothèses théoriques et de cadres de référence déclinés systématiquement sur un grand nombre de cas d'applications. La recherche en gestion est davantage plurielle, ce qui nuit peut-être à sa lisibilité. Mais elle a profondément renouvelé ses approches et ses prescriptions en s'intéressant notamment aux phénomènes cognitifs, aux activités et aux raisonnements de conception, à partir d'une épistémologie de l'action collective (Hatchuel, 2012; Martinet et Pesqueux, 2013) qui n'a pas d'équivalent dans la pensée économique standard qui reste attachée à un paradigme de l'information et de la décision. Le rapport à l'empirie constitue la deuxième différence fondamentale : la modélisation sous forme de faits stylisés permet de s'affranchir, au moins partiellement, de critères de pertinence et de fidélité dans la restitution des faits empiriques. Le souci d'une robustesse empirique conduit les recherches qualitatives en gestion, à l'instar de celles dans d'autres disciplines des sciences sociales qui partagent ce même souci (histoire, sociologie, anthropologie, économie politique, etc.), à développer des analyses plus fines, mais également plus ambiguës, dont les prescriptions apparaissent moins générales car plus contingentes aux situations étudiées.

La question n'est pas ici de rejeter le principe de modélisation qui constitue un mode de raisonnement tout à fait pertinent pour traiter de phénomènes complexes. Mais il n'est pas inutile de rappeler que tout exercice de modélisation s'appuie sur une doctrine implicite qui n'est jamais neutre et dont il convient d'interroger les fondements et les effets. À cet égard, l'orientation normative et instrumentale de la théorie des incitations, telle qu'elle est incarnée par les travaux de Jean Tirole, n'est pas sans soulever de nombreuses questions. Sumantra Goshal s'interroge, par exemple, sur les dégâts que produisent les cours sur la gouvernance d'entreprise où, à partir de la théorie de l'agence, l'on enseigne à des générations d'étudiants et de managers que l'on ne peut faire confiance aux managers et qu'il s'agit d'aligner leurs intérêts sur ceux des actionnaires (Goshal, 2005). L'orientation instrumentale de cette théorie, où les problèmes d'action collective sont censés être résolus par le biais de systèmes d'incitation, se fonde, en effet, sur le présupposé d'un alignement mécanique des comportements des agents en fonction de ces incitations. Cela revient, en quelque sorte, à nier les capacités réflexives, cognitives et critiques des acteurs et leur capacité à détourner les systèmes d'évaluation, aussi sophistiqués fussent-ils. Ce ne sont pourtant pas les exemples de tels détournements qui manquent, comme l'ont montré depuis longtemps les travaux sur les instruments de gestion (voir Berry, 1983 ; Moisdon, 1997 ; Aggeri et Labatut, 2010 ; Vaujany (de), 2006). 
Quand les incitations sont perçues comme injustes ou violentes, quand elles sont mises en place sans discernement au nom d'une norme discutable, à l'instar de certaines pratiques de gouvernance d'entreprise, les phénomènes de résistance et de contournement ne sont pas le symptôme d'une déviance mais, au contraire, un signe de vitalité, voire, dans certains cas, une condi- tion de survie. Ils ne sont, au fond, que la manifestation d'une volonté d'être acteur plutôt qu'agent. Car, en effet, que seraient une société, une organisation, une entreprise ou un projet collectif où chacun soupçonnerait son proche, son voisin ou son collègue d'opportunisme, chercherait à lui cacher des informations et à exercer visà-vis de lui des relations d'agence ?

\section{BIBLIOGRAPHIE}

Adant I., Godard O. et Hommel T. (2005). «Expertise scientifique et gestion de la contestabilité sociale en présence d'acteurs à visées stratégiques », Cahier de recherche du laboratoire d'économétrie, école Polytechnique, $\mathrm{n}^{\circ} 16$.

Aggeri F. et Labatut J. (2010). «La gestion au prisme de ses instruments. Une analyse généalogique des approches théoriques fondées sur les instruments de gestion », Finance Contrôle Stratégie, vol. 13, n 3, septembre, p. 5-37.

Aggeri F., Pezet E., Abrassart C. et Acquier A. (2005). Organiser le développement durable, Vuibert, Paris.

Aggeri F. (2000). «Les politiques d'environnement comme politiques de l'innovation », Gérer et Comprendre, juin, p. 31-43.

Aggeri F. (1999). "Environmental policies and innovation: a knowledge-based perspective on cooperative approaches", Research Policy, vol. 28, n 7, p. 699-717.

Akerlof G. (1970). "The market for 'Lemons': Quality uncertainty and the market mechanism”, Quarterly Journal of Economics, vol. 84, n³ 3, p. 488-500.

Argyris C. et Schön D. (1978). Organizational learning, Addison-Wesley, Mass.

Barnard C. (1938). The functions of the executive, Harvard University Press.

Benabou R. et Tirole J. (2010). "Individual and corporate social responsibility”, Economica, 77, p. 1-19.

Benkeltoum N. (2011). Gérer et comprendre l'open source. Une modélisation en termes de "régimes », Presse des Mines.

Berry M. (1983). Une technologie invisible. L'impact des instruments de gestion sur l'évolution des systèmes humains, publication de l'École polytechnique.

Blair M. et Stout L. (1999). "A team production theory of corporate law", Virginia Law Review, vol. 85, n², p. 248-290.

Brès L. et Gond J.P. (2014). "The visible hand of consultants in the construction of the markets for virtue: translating issues, negotiating boundaries and enacting responsive regulations", Human Relations, May, p. 1-36. 
Capron M. et Quairel F. (2015). L'entreprise dans la société. Une question politique, La Découverte.

Charreaux G. et Desbrières P. (1998). « Gouvernance des entreprises : valeur partenariale contre valeur actionnariale », Finance - Contrôle - Stratégie, vol. 1, n 2, juin 1998, p. $57-88$.

Encaoua D. (2015). « Pouvoir de marché, stratégies et régulation : Les contributions de Jean Tirole, prix Nobel d'économie $2014 »$, Revue d'économie politique, vol. 125, n 1, p. 1-76.

Favereau O. (2006). « Objets de gestion et objet de la théorie économique », Revue française de gestion, vol. $32, \mathrm{n}^{\circ} 160$, p. 67-79

Gawer A. et Cusumano M. (2010). "Industry platform and ecosystem innovation", Journal of Product Innovation Management, 31, p. 417-433.

Godard O. (2009). «Quelle architecture internationale pour la politique climatique ? Les fausses évidences du rapport Tirole ", II. Ajustement aux frontières, enchères et transferts Nord-Sud, document de travail, École polytechnique.

Gomez P.Y. (2003). « Jalons pour une histoire des théories du gouvernement des entreprise », Finance Contrôle Stratégie, vol. 6, n 4, décembre, p. 183-208.

Ghoshal, S. (2005). "Bad management theories are destroying good management practices", Academy of Management learning \& education, vol. 4, $n^{\circ}$ 1, p. 75-91.

Grossman S. et Hart O. (1986). "The costs and benefits of ownership: a theory of vertical and lateral integration”, Journal of Political Economy, 94, p. 691-719.

Hatchuel A. (1994). « Frédéric Taylor : une lecture épistémologique. L'expert, le théoricien, le doctrinaire ", L'invention de la gestion, Histoire et pratiques, Bouilloud J.P. et Lecuyer B.P. (éd.), L'Harmattan, p. 68-95.

Hatchuel A. (2012). «Quel horizon pour les sciences de gestion?», Les nouvelles fondations des sciences de gestion, David A., Hatchuel A. et Laufer R., Presses des mines, Paris, p. 17-41.

Laffont J.J. et Tirole J. (1991). "The politics of government decision making: A Theory of regulatory capture”, Quarterly Journal of Economics, 106, p. 1089-1127.

Lerner J. et Tirole J. (2002). "Some simple economics of open source", Journal of Industrial Economics, vol. 50, n², p.197-234.

Martinet A.C., Pesqueux Y. (2013). Épistémologie des sciences de gestion, Vuibert.

Martinet A.C. (1984). Management stratégique : organisation et politique, McGraw-Hill.

Matten J. et Moon J. (2008). "Implicit and explicit CSR: a conceptual framework for a comparative understanding of corporate social responsibility", Academy of Management Review, vol. $33 \mathrm{n}^{\circ} 2$, p. 404-424.

Moisdon J.C. (sous la dir.) (1997). Du mode d'existence des outils de gestion, Séli Arslan.

Muniesa F. et Callon M. (2008). "La performativité des sciences économiques", Cahier de recherche $d u$ CSI, Mines ParisTech, $\mathrm{n}^{\circ} 10$. 
Nonaka I. et Takeuchi H. (1995). The Knowledge Creating Company: How Japanese Companies Create the Dynamics of Innovation, Oxford University Press, New York.

Peaucelle J.L. (2003). Henri Fayol, Inventeur des outils de gestion. Textes originaux et recherches actuelles, Economica.

Porter M.E. et Van der Linde C. (1995). "Toward a new conception of the environmentcompetitiveness relationship", Journal of economic perspectives, vol. 9, n 4, p. 97-118.

Porter M. et Kramer (2006). "The link between competitive advantage and corporate social responsibility", Harvard Business Review, vol. 84, n 12, p. 78-92.

Rochet J.C. et Tirole J. (2003). "Platform competition in two-sided markets", Journal of the European Economic Association, vol. 1, n 4, p. 990-1029.

Segrestin B. et Hatchuel A. (2012). Refonder l'entreprise, la République des idées.

Stigler G. (1971). "The economic theory of regulation", Bell Journal of Economics, vol. 2, p. 3-21.

Tirole J. (2013). «Rationalité, psychologie et économie », Revue Française d'Économie, vol. XXVIII, p. 9-33.

Tirole J. (2001). “Corporate Governance”, Econometrica, vol. 69, n 1, p. 1-35.

Vaujany F.X. (de) (2006). « Pour une théorie de l'appropriation des outils de gestion : vers un dépassement de l'opposition conception-usage », Management \& Avenir, p. 109-126.

Von Krogh G. et Von Hippel E. (2003). “Open source software: Introduction to a special issue of research policy”, Research Policy, vol. 32, n 7, July, p. 1149-57. 
